\title{
The Response of Skeletal Muscle-Expressed Neurotrophins to Acute Resistance Exercise in Male Wistar Rats
}

\author{
${ }^{1}$ Rasoul Eslami* ${ }^{2}$ Reza Gharakhanlou, ${ }^{3}$ Abdol-Hossein Parnow \\ ${ }^{1}$ Faculty of Sport Sciences, Allameh Tabataba'i University, Tehran, Iran. ${ }^{2}$ Department of Exercise Physiology, Faculty of \\ Humanity, Tarbiat Modares University, Tehran, Iran. ${ }^{3}$ Faculty of Sport Sciences, Razi-e-Kermanshah University, \\ Kermanshah, Iran.
}

\begin{abstract}
Background. BDNF and NT-4/5 have been proposed to be involved in the coordinated adaptations of the neuromuscular system to the elevated level of activity, but an activity-dependent expression of neurotrophins in skeletal muscle is not well established. Objectives. We, therefore, investigated the effect of one session of resistance exercise on mRNA expression of some neurotrophins in Slow and fast muscles of Wistar rats. Methods. The resistance training protocol consisted of climbing a 1-meter-long ladder, with a weight attached to a tail sleeve. Twenty-four hours following the main training session, Soleus and Flexor Hallucis Longus (FHL) muscles were removed. mRNA expression of BDNF, NT4/5, TrkB and p75 proteins was assessed by Quantitative RT-PCR. Results. The data analysis showed that one session of resistance exercise significantly $(\mathrm{p}<0.05)$ decreased mRNA expression of NT4/5 in soleus muscle, but not in FHL muscle. No significant effects of one resistance exercise bout were detected for BDNF and trkB. Our results also show that p75 mRNA levels in the soleus muscle were significantly elevated (7folds) after one resistance training bout $(\mathrm{p}<0.05)$. Conclusion. The results indicate differential control of BDNF and NT-4/5 expression following resistance exercise in skeletal muscle. Also, we have provided evidence supporting the role of the p75 receptor in neurotrophins response to resistance exercise as a mechanical stimulus.
\end{abstract}

KEY WORDS: Brain-Derived Neurotrophic Factor, Neurotrophin-4/5, Tropomyosin-Related Kinase B, P75 Receptor, Resistance Training, Skeletal Muscle.

\section{INTRODUCTION}

Although the effects of increased activity on mammalian skeletal muscle have been studied extensively, evidence that innervating how motoneuron and muscle interact in response to increased activity is sparse $(1,2)$. For example, the question of how skeletal muscle as a classic target tissue regulates survival of spinal motoneurons and modulate neuromuscular transmission during activity has been one of the major topics in exercise physiology (3).
There is some suggestion that trophic factors are critical modifiers of the structure and function of neuromuscular networks $(1,2)$. However, the mechanisms for how trophic factors impacts neuromuscular function at the cellular and molecular levels are largely unexplored. Two muscle-derived neurotrophin proteins, brainderived neurotrophic factor (BDNF) and neurotrophin-4/5 (NT-4/5), have been proposed to be involved in the coordinated adaptation of the

*. Corresponding Author:

Rasoul Eslami

E-mail: eslami.rasul@gmail.com 
neuromuscular system to elevated activity $(4,5,6)$. The physiological significance of changes in neurotrophin expression is contingent on the availability of appropriate signal transduction receptors (7). The functions of the neurotrophins are effected via two classes of receptor: the Trk (tropomyosin receptor kinase) family of RTKs (receptor tyrosine kinases) and p75NTR (p75 neurotrophin receptor) $(8,9)$.

To date, a few studies have performed on muscle-derived neurotrophins expression after physical activity. Recently, brief treadmill training bouts over 5 days were found to produce large increases in BDNF mRNA in soleus muscle $(2,6,10)$, but one treadmill exercise bout do not effect on BDNF expression (2). No significant effects were detected for NT-4/5 and trkB in soleus following 5 or 10 days of exercise. In addition, there was no effect of 5 or 10 days of exercise on BDNF, NT-4/5, and trkB in medial gastrocnemius (6). Although another neurotrophin receptor, p75, might be more relevant to their role in skeletal muscle (6), there is not any research that had studied effect of exercise training on p75 in skeletal muscle.

This data was demonstrated that effects of exercise training on neurotrophins expression in skeletal muscle are not clear.

Consequently, we utilized different type of exercise training, resistance training, to study effect of increased activity on neurotrophins expression in skeletal muscle. As BDNF and NT$4 / 5$ can initiate intracellular signalling through the same cell surface receptor, tropomyosin-related kinase B (trkB) $(6,11,12)$, it is of interest that they have evaluated together following similar exercise conditions. Moreover, we add p75 to elucidate whether a functional relationship between BDNF, NT-4/5 and p75 exists in adult skeletal muscle.

\section{MATERIALS AND METHODS}

Animals. Sixteen adult, male Wistar rats (1315 week of age) were obtained from the Pasture Institute (Tehran, Iran) and maintained in the Animal House, School of Medical Sciences of Tarbiat Modares University (TMU). All animals were housed under similar living conditions in plastic cages with volume of $46 \mathrm{~L}$. The light-dark cycle was $12 \mathrm{~h}$. Temperature was $22 \pm 1.4{ }^{\circ} \mathrm{C}$, and humidity was $55.6 \pm 4.0 \%$. Animals were fed with a pellet rodent diet and had free access to water. Animals were randomly assigned into two groups including: sedentary (SED) $(n=8)$ and resistance exercise (RE) $(n=8)$. Ethics Committee of TMU approved the experimental protocol, and their Guidelines for Care and Use of Laboratory Animals were followed.

Resistance Exercise (RE). The animals were required to climb a vertical ladder inclined at 85 degrees with weights appended to their tail. There were 26 rungs across the $1 \mathrm{~m}$ ladder. The animals were positioned to ensure that they performed each sequential step, where one repetition along the ladder required 26 total lifts by the animal (or 13 lifts per limb) (13). All animals were weighed to determine the amount of mass to append to their tails. The weight attached was $30 \%$ of their body weight of each resistance exercised animals. The resistance exercise consisted of 3 sets of 5 repetitions with a $1 \mathrm{~min}$ rest interval between the reps and 2 minutes between the sets for one session (14). The control animals were handled on the same days and times as the trained groups in order to minimize any stress attributable to handling (13).

Tissue Preparation. Since previous study show that peak point of BDNF expression after acute exercise was 24-48 h later (10), Twentyfour hours following the main training session, animals were anaesthetized with a mixture of Ketamine (75mg/kg-1) and Xylazine ( $5 \mathrm{mg} / \mathrm{kg}-1)$ which was administered intraperitoneally. The soleus (SOL, as ST muscle) and Flexor Hallucis Longus (FHL, as FT muscle) $(15,16)$ were surgically extracted under sterile, RNase-free conditions on ice and immediately fresh frozen in liquid nitrogen. All tissue was stored at $-80^{\circ} \mathrm{C}$ until further processing.

RNA Isolation and real-time quantitative $R T$ $\boldsymbol{P C R}$. All frozen muscles were homogenized with an RNase-free mortar and pestle cooled in liquid nitrogen and dry ice. Total RNA was isolated from the tissue using Trizol reagent (Invitrogen), which was performed as per the manufacturer's specifications. The concentration and purity of the total RNA sample was determined by ultraviolet (UV) spectrophotometer at $260 \mathrm{~nm}$ and 280 nm (ND-1000; Nanodrop).

Total RNA preparations were treated with DNase I (Takara) prior to reverse transcription to eliminate genomic DNA following RNA 
isolation. Reverse transcription was performed with cDNA synthesis kit (PrimeScript RT Reagent Kit, Takara) as per the manufacturer's recommendations.

Real-time PCR for all genes of interest was performed with the 7500 Fast Thermocycler (Applied Biosystems, version 2.0.1) with SYBR Green Master Mix (Takara). The real-time PCR reaction included the following: 200-400nM primers, 5 ng cDNA samples and $1 \mathrm{X}$ SYBR
Green Master Mix. A reaction volume of $20 \mu 1$ was run on a sealed optical plate in triplicate as per the manufacturer's recommendations. The nucleotide sequences of sense and antisense primers is shown in table 1. Relative quantification of target gene expression was performed using the comparative CT method as described in detail elsewhere (17). To quantify the levels of the target gene expression, the formula $2^{\wedge}(-\Delta \Delta \mathrm{ct})$ was used.

Table 1. The nucleotide sequences of sense and antisense primers.

\begin{tabular}{ccc}
\hline Gene & Sense & Antisense \\
\hline GAPDH & GAACATCATCCCTGCATCCA & CCAGTGAGCTTCCCGTTCA \\
\hline$\beta$-Actin & TCAGGTCATCACTATCGGCAATG & GCATAGAGGTCTTTACGGATGTCAAC \\
\hline NT-4/5 & CCCTGCGTCAGTACTTCTTCGAGAC & CTGGACGTCAGGCACGGCCTGTTC \\
\hline BDNF & CGACGTCCCTGGCTGACACTTTT & AGTAAGGGCCCGAACATACGATTGG \\
\hline TrkB & CGACGTCCCTGGCTGACACTTTT & AGTAAGGGCCCGAACATACGATTGG \\
\hline p75 & CAACGGTCAGAACGGAGCATC & AGAGGGTGGTCAGAAGCAAGG
\end{tabular}

Statistical Analyses. We used $2^{-\Delta \Delta \mathrm{CT}}$ method to analyze raw data from real-time quantitative PCR. Overall Differences between control and experimental groups were determined using an Independent-t test. The level of significance set was at $\mathrm{P}<0.05$ for all statistical comparisons and the results were expressed as the mean \pm standard deviation $(\mathrm{SD})$.

\section{RESULTS}

Effects of resistance exercise on control genes. Relative quantification of gene expression is dependent on the use of control genes for standardization of experimental data to control for shifts in mRNA levels that could be produced by the experimental intervention (6). There was a main effect on expression of $\beta$-Actin with resistance exercise in both soleus and FHL muscles (respectively, $\mathrm{p}=0.099 ; \mathrm{p}=0.038$ ) (Fig. 1). However, Expression of GAPDH was most stable following resistance exercise in both soleus and FHL muscles $(\mathrm{p}=0.811$ and $\mathrm{p}=0.576$; respectively) (Fig. 1), therefore, was selected to normalize experimental data investigating the effects of resistance exercise on neurotrophins expression in skeletal muscle.

Effects of resistance exercise on BDNF, NT$4 / 5$, TrkB and $p 75$. It was difficult to detect mRNA of BDNF in both soleus and FHL muscle. The mRNA of BDNF detected in only three or four samples for each group. However, there were no detectable changes in the level of BDNF mRNA in soleus and FHL muscles after one session of resistance exercise (fig. 2). Data show that NT-4/5 mRNA was decreased significantly in soleus muscle $(p=0.003)$ following resistance training but it was not changed in FHL muscle $(\mathrm{p}=0.743)$ (Fig. 2).

Our results also indicate that the TrkB mRNA level was 2fold higher following resistance training in both muscles when compared with control group. Despite TrkB mRNA level was increased after resistance training, this increase was not statistically significant (soleus: $\mathrm{p}=0.392$; FHL: $\mathrm{p}=0.251$ ) (Fig. 3). One session of resistance training also increased levels of p75 mRNA in soleus ( 7.5 fold) and FHL ( 2.8 fold) muscles. This increase was significant for soleus muscle and was not significant for FHL muscle ( $\mathrm{p}=0.043$ and $\mathrm{p}=0.417$; respectively) (Fig. 3).

\section{DISCUSSION}

Effects of resistance Exercise on BDNF Expression. Previous studies indicated that neurotrophins in motor-nervous system are generated in excess, and that modulating the interrelation of neuromuscular system (18). Some of neurotrophins, as BDNF and NT-4/5, are produced in skeletal muscle in fewer amounts. In our study we detected BDNF mRNA in low content that limited 
us to data analysis. Also, other studies reported low level of BDNF mRNA in developing and postnatal rodent skeletal muscle (18-23). In addition, some studies could not detect any BDNF expression in developing muscle of mice and rats $(24,25)$. It is clear that precise and novel methods are required for accurate measurement of BDNF expression in skeletal muscle.

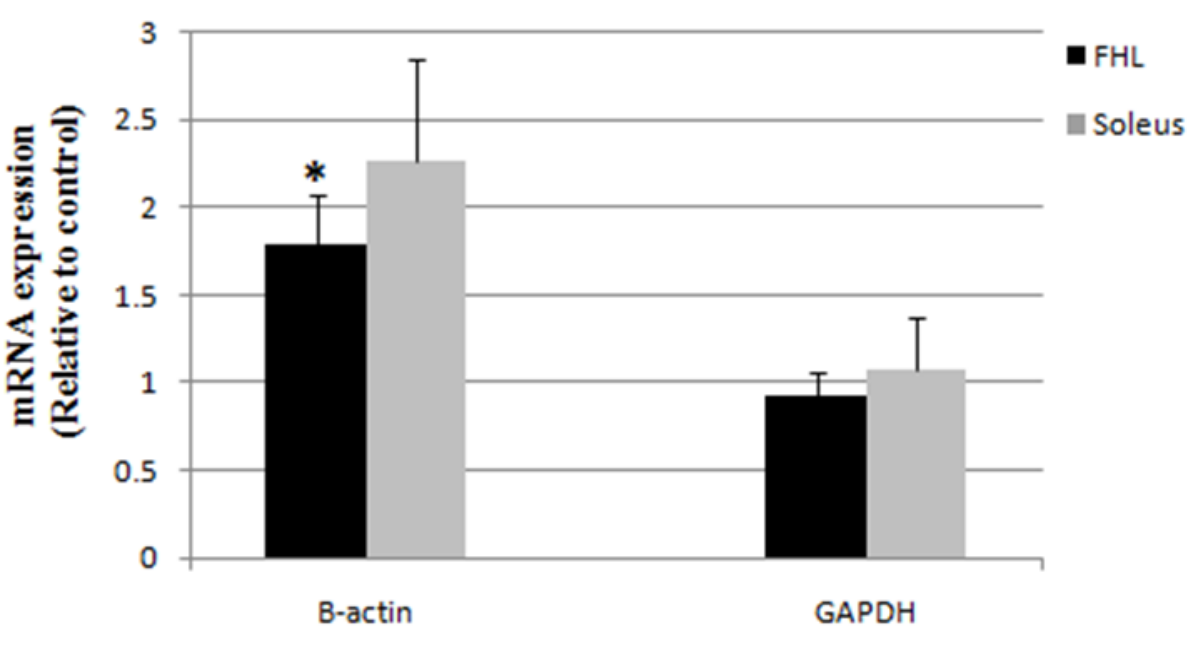

Figure 1. Effects of one session of resistance exercise on $\beta$-actin and GAPDH expression in Flexor Hallucis Longus (FHL) (filled bars) and soleus (open bars). Values represent mean \pm standard deviation and are expressed as a percentage of control expression levels. Asterisk denotes statistical significance between exercise and control groups $(\mathrm{p}<0.05)$.

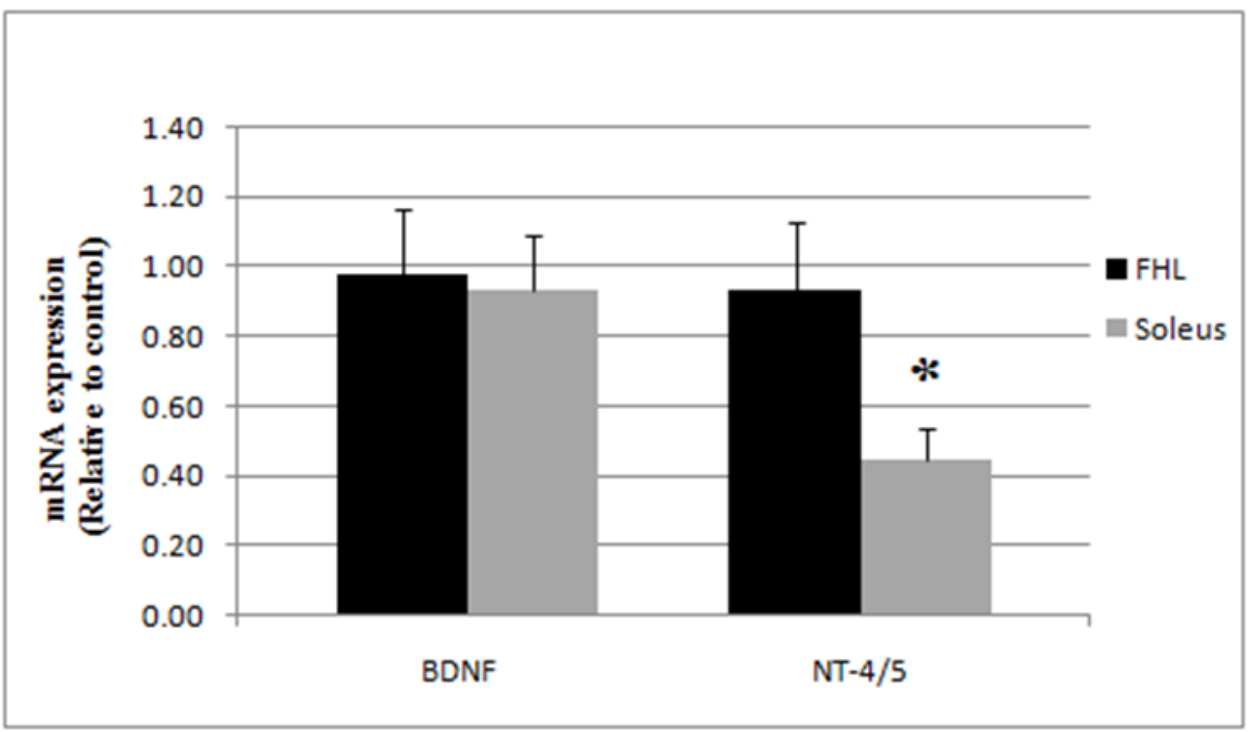

Figure 2. Effects of one session of resistance exercise on BDNF and NT-4/5 expression in Flexor Hallucis Longus (FHL) (filled bars) and soleus (open bars). Values represent mean \pm standard deviation and are expressed as a percentage of control expression levels. Asterisk denotes statistical significance between exercise and control groups $(\mathrm{p}<0.05)$. 


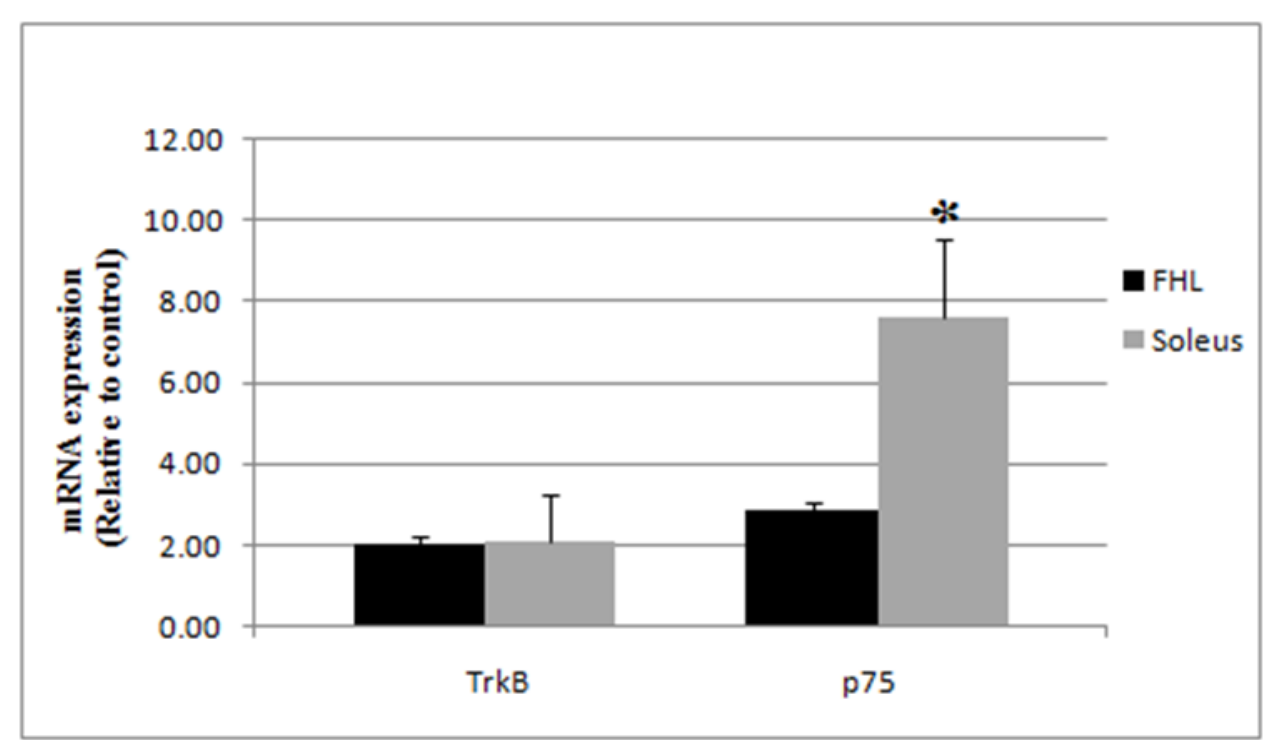

Figure 3. Effects of one session of resistance exercise on TrkB and p75 expression in Flexor Hallucis Longus (FHL) (filled bars) and soleus (open bars). Values represent mean \pm standard deviation and are expressed as a percentage of control expression levels. Asterisk denotes statistical significance between exercise and control groups $(p<0.05)$.

Despite the difficulty of BDNF expression in skeletal muscle, our study indicates that one resistance exercise bout did not result in any change in BDNF expression in soleus or FHL muscle. Although muscle-derived BDNF was identified as an activity-dependent factor, the most previous studies did not reveal effect of single bout of exercise on BDNF expression $(2,10)$. However, a robust increase in BDNF mRNA was observed after repetitive exercise training only in soleus muscle $(2,6,7,10)$, while, there is not any study in that BDNF mRNA alteration was reported following exercise training in other type of skeletal muscle. Therefore, repetitive training over a period of days apparently can sustain elevated levels of BDNF mRNA for a longer period of time than that elicited by a single bout of exercise.

It is becoming well-established that BDNF have a role in the development of functional connectivity between skeletal muscle and the spinal cord (2). For example, it has been hypothesized that skeletal-muscle-derived BDNF enhances the survival of innervating motor neurons throughout their lifespan and potentiates neuromuscular transmission (26,27). This hypothesis is supported by several lines of evidence including, for example, the expression of BDNF in skeletal muscle and retrograde transport of exogenously applied BDNF to distinct motor neuron cell bodies (27-29). Therefore, it appears that sufficient stimulation and time is required for activity-dependent effects of muscle-derived BDNF. Since our results show that one resistance exercise bout did not result in any change in BDNF expression, it seems likely that one bout of resistance stimulation is not sufficient to induce BDNF expression signalling in skeletal muscle. In addition, BDNF may exert these effects via its receptors as TrkB and p75.

Effects of Exercise on NT-4/5 Expression. Activity-dependent expression of NT-4/5 mRNA has been demonstrated through decreased expression following denervation and neuromuscular transmission blockade with abungarotoxin and increased expression following electrical stimulation of the sciatic nerve $(6,28,30)$. Nonetheless, Deschenes et al. could not to establish the activity-dependent expression of NT-4/5 in soleus muscle. They reported that the stimulus of unloading (hindlimb suspension) was inadequate to alter NT-4 content in soleus muscle (31). Results of the Ogborn study agree with this finding and indicate that exercise of sufficient intensity to increase expression of BDNF in soleus has no effect on NT-4/5 mRNA in soleus and medial gastrocnemius (6). 
To date, no study reported effects of exercise training on NT-4/5mRNA in skeletal muscle. Only, Sakuma et al. reported decreased level of NT-4/5 protein following mechanical overloading in plantaris muscle (24). To our knowledge, our study is the first to show that increase level of activity as one resistance exercise bout result in NT-4/5 mRNA decrease in soleus muscle, wherever, it did not show any change in FHL muscle. Carrasco et al. have provided evidence demonstrating that NT-4/5 is required for the normal development of the slow muscle fiber phenotype of the rat SOL. They believed that muscle-derived NT-4/5 probably forms or activates a retrograde signal that influences the pattern of activity of SOL motoneurons and, via this mechanism, leads to changes that promote the upregulation of the slow MyHC isoform in the SOL muscle (32). On the other hand, it has been established that resistance training could shift MyHC isoforms to IIA in skeletal muscle (33). Therefore, the significant decrease of NT-4/5 mRNA was due to the fact that resistance exercise stimulates signalling of transition of MyHC to IIA in soleus muscle.

It has been show that NT-4/5 promotes sciatic nerve sprouting (28), induces postsynaptic potentiation (34), inhibits agrin-induced AChR clustering (35), and induces mitochondrial proliferation (36). Mechanical overloading, bupivacaine injection-induced regeneration and denervation markedly change the activity and/or volume of muscle fiber (24). Structural and contractile proteins as well as muscle-specific enzymes needed in hypertrophied muscle may be upregulated on stimulation of activity-dependent postsynaptic potentiation of NT-4. It is possible that NT-4/5, which inhibits agrin-induced AChR clustering, is lost in the early phase of regeneration due to reconstruction of neuromuscular junctions after resistance exercise. Administering BDNF, NT-3 or NT-4 in combination with CNTF at the site of nerve transection reduces motor neuron loss and prevents axotomy-induced muscle atrophy $(29,37)$. In adults, NT-4 supports slow type of motor units in the soleus muscle, preventing muscle atrophy and muscle fiber loss $(37,38)$ However, no study has yet determined the adaptive response of NT-4/5 in hypertrophied and regenerating muscle (24). However, the effects of exercise on the expression of $\mathrm{NT}-4 / 5$ remain poorly understood.

Effects of Exercise on TrkB and p75 Expression. The physiological significance of changes in neurotrophin expression is contingent on the availability of appropriate signal transduction receptors (7). BDNF and NT-4/5 can initiate intracellular signalling through the same cell surface receptor, TrkB and p75. In this study, we have investigated this ligand and their receptors together for first time. Our results show that TrkB mRNA increased 2folds following one resistance training bout in both soleus and FHL muscle, but this increase has not been statistically significant. Although, few data currently exist on TrkB expression in skeletal muscle, all of them are agree with us results. Recently, Ogborn et al. found that 5 or 10 days of treadmill exercise did not result in any appreciable change in $\operatorname{trkB}$ expression in medial gastrocnemius or soleus (6). Also, Gomez-Pinilla et al. found 7 days of voluntary wheel running could not elevate trkB expression in soleus; however, trkB mRNA levels in the exercised rats were higher than control after 3 days of exercise (7).

Although changes in expression of BDNF mRNA often occur in parallel with changes in trkB expression (7), recent data suggest that another neurotrophin receptor, p75, might be more relevant to BDNF's role in skeletal muscle (6). It was showed that Trk receptors appear at the cell membrane mostly in a complex with p75NTR. When P75 co-expressed with Trk receptor, exert a positive regulatory effect on Trk functions $(3,39,40)$. Mousavi and Jasmin proposed that p75 receptor is a satellite cell marker and strong regulator for post-injury regeneration in skeletal muscle (41). Deponti et al. indicated that NGF acts through its lowaffinity receptor p75NTR in a developmentally regulated signaling pathway necessary to myogenic differentiation and muscle repair in vivo (42). Our results show that p75 mRNA levels in the soleus muscle were significantly elevated (7folds) after one resistance training bout; In addition, p75 mRNA was trend to increase in FHL muscle. Since, in this study, we used resistance stimulus as a mechanical overload and it could activate myogenic pathways, it is likely possible that elevated p75 expression are the result of post-exercise remodeling in skeletal 
muscle. It is also possible that these effects are the result of the change in $\mathrm{TrkB} / \mathrm{p} 75$ ratio, and the resultant change in function of $\mathrm{p} 75$ from adjunctive to TrkB to independently signalling receptor. However, Colombo et al. indicated that p75 is upregulated on skeletal myofibres in inflammatory myopathies in vivo and promotes resistance to inflammatory mediators in vitro suggest that neurotrophin signalling through p75 may mediate a tissue-protective response to inflammation in skeletal myofibres (43). Therefore, increase level of p75 mRNA may be as result of muscle inflammation following resistance exercise.

\section{CONCLUSION}

In order to proven activity-dependent role of muscle-derived neurotrophins, we utilized resistance training as a mechanical overload. We have demonstrated that one resistance training bout did not result in any change in BDNF expression in soleus or FHL muscle. This study indicated a significant decrease of NT-4/5mRNA levels in soleus muscle in exercise group, while there was no any change in FHL muscle. Consequently, we have provided evidence supporting role of $\mathrm{p} 75$ receptor in neurotrophins response to resistance exercise as a mechanical stimulus. In summary, the functional significance of muscle-derived neurotrophins in the adaptation of skeletal muscle to exercise is poorly understood and future work is required to determine whether NT-4/5 even plays a role in neuromuscular adaptations to altered activity levels.

\section{APPLICABLE REMARKS}

- It seems that neurotrophins and their receptors are main factors in muscle response to resistance exercise as a mechanical stimulus.

- Increased activity as resistance training has a potential for skeletal muscle remodeling via alteration in neurotrophins expression.

\section{REFERENCES}

1. Beaumont E, Gardiner PF. Endurance training alters the biophysical properties of hindlimb motoneurons in rats. Muscle Nerve 2003; 27: 228-236.

2. Gomez-Pinilla F, Ying Z, Opazo P, Roy RR, Edgerton VR. Differential regulation by exercise of BDNF and NT3 in rat spinal cord and skeletal muscle. Eur J Neurosci 2001;13:1078-1084.

3. Chevrel G, Hohlfeld R, Sendtner R. The role of neurotrophins in muscle under physiological and pathological conditions. Muscle Nerve 2006; 33: 462-476.

4. Munson JB, Foehring RC, Mendell LM, Gordon T. Fast-to-slow conversion following chronic low-frequency activation of medial gastrocnemius muscle in cats II. Motoneuron properties. J Neurophysiol 1997;77:2605-2615.

5. Beaumont E, Gardiner PF. Endurance training alters the biophysical properties of hindlimb motoneurons in rats. Muscle Nerve 2003;27: 228-236.

6. Ogborn DI, Gardiner PF. Effects of exercise and muscle type on BDNF, NT-4/5, and TrkB expression in skeletal muscle. Muscle Nerve 2010; 41: 385-391.

7. Gomez-Pinilla F, Ying Z, Roy RR, Molteni R, Edgerton VR. Voluntary exercise induces a BDNF-mediated mechanism that promotes neuroplasticity. J Neurophysiol 2002; 88: 2187-2195.

8. Thomas SM, DeMarco M, D'Arcangelo G, Halegoua S, Brugge JS. Ras is essential for nerve growth factor- and phorbol ester-induced tyrosine phosphorylation of MAP kinases. Cell. 1992; 68(6):1031-40.

9. Hennigan A, O'Callaghan RM, Kelly AM. Neurotrophins and their receptors: rolesin plasticity, neurodegeneration an neuroprotection. Biochemical Society Transactions 2007; 35: 424-427.

10. Cuppini R, Sartini S, Agostini D, Guescini M, Ambrogini P, Betti M, et al. BDNF expression in rat skeletal muscle after acute or repeated exercise. Arch Ital Biol 2007;145:99-110.

11. Klein R, Lamballe F, Bryant S, Barbacid M. The trkB tyrosine protein kinase is a receptor for neurotrophin-4. Neuron 1992;8:947-956.

12. Klein R, Nanduri V, Jing SA, Lamballe F, Tapley P, Bryant S, et al. The trkB tyrosine protein kinase is a receptor for brain-derived neurotrophic factor and neurotrophin-3. Cell 1991;66:395-403.

13. Godfrey JK, Kayser BD, Gomez GV, Bennett J, Jaque SV, Sumida KD. Interrupted resistance training and BMD in growing rats. Sports Med 2009; 30: $579-584$. 
14. Lee $\mathrm{S}$ and Farrar RP. Resistance training induces muscle-specific changes in muscle mass and function in rat. JEP online 2003; 6(2):80-87.

15. Forsgren S, Bergh A, Carlsson E, and Thornell LE. Calcitonin gene-related peptide expression at endplates of different fibre types in muscles in rat hind limbs. Cell and Tissue Research 1993; 274(3): 439-446.

16. Fontaine B, Klarsfeld A, Hokfeld T, and Changeux JP. Calcitonin gene-related peptide, a peptide present in spinal cord motoneurons, increases the number of acetylcholine receptors in primary cultures of chick embryo myotubes. Neuroscience Letters 1986; 71: 59-65.

17. Kenneth JL and Thomas DS. Analysis of Relative Gene Expression Data Using Real-Time Quantitative PCR and the $2^{-\Delta \Delta C T}$ Method. Methods 2001; 25: 402-408.

18. Sakuma K, and Yamaguchi A. The Recent Understanding of the Neurotrophin's Role in SkeletalMuscle Adaptation. Journal of Biomedicine and Biotechnology 2011; doi:10.1155/2011/201696.

19. Griesbeck O, Parsadanian AS, Sendtner M, and Thoenen H. Expression of neurotrophins in skeletal muscle: quantitative comparison and significance for motoneuron survival and maintenance of function. Journal of Neuroscience Research 1995; 42: 21-33.

20. Ip FCF, Cheung J, and Ip NY. The expression profiles of neurotrophins and their receptors in rat and chicken tissues during development," Neuroscience Letters 2001; 301( 2): 107-110.

21. Koliatsos VE, Cayouette MH, Berkemeier LR, Clatterbuck R E, Price DL, and Rosenthal A. Neurotrophin $4 / 5$ is a trophic factor for mammalian facial motor neurons. Proceedings of the National Academy of Sciences of the United States of America 1994; 91( 8): 3304-3308.

22. Koliatsos VE, Clatterbuck RE, Winslow JW, Cayouette MH, and Price DL. Evidence that brain-derived neurotrophic factor is a trophic factor for motor neurons invivo. Neuron 1993; 10( 3): 359-367.

23. Maisonpierre PC, Belluscio L, Friedman B et al. NT-3, BDNF, and NGF in the developing rat nervous system: parallel as well as reciprocal patterns of expression," Neuron 1990; 5(4): 501-509.

24. Sakuma K, Watanabe K, Sano M, Uramoto I, Nakano H, Li YJ, et al. A possible role for BDNF, NT-4 and TrkB in the spinal cord and muscle of rat subjected to mechanical overload, bupivacaine injection and axotomy. Brain Res 2001; 907: 1-19.

25. Schecterson LC and Bothwell M. Novel roles for neurotrophins are suggested by BDNF and NT-3 mRNA expression in developing neurons," Neuron 1992; 9(3): 449-463.

26. Oppenheim RW, Qin-Wei Y, Prevette D, and Yan Q. Brain-derived neurotrophic factor rescues developing avian motoneurons fromcell death. Nature1992; 360(6406): 755-757.

27. Zhang XH and Poo MM. Localized synaptic potentiation by BDNF requires local protein synthesis in the developing axon. Neuron 2002; 36 (4): 675-688.

28. Funakoshi H, Belluardo N, Arenas E, Yamamoto Y, Casabona A, Persson H and Ibanez CF. Musclederived neurotrophin-4 as an activity-dependent trophic signal for adult motor neurons. Science 1995; 268: 1495-1499.

29. Mousavi K, Parry DJ, and Jasmin B J. BDNF rescues myosin heavy chain IIB muscle fibers after neonatal nerve injury. American Journal of Physiology 2004; 287: C22-C29.

30. Funakoshi H, Frisen J, Barbany G, Timmusk T, Zachrisson O, Verge VM, et al. Differential expression of mRNAs for neurotrophins and their receptors after axotomy of the sciatic nerve. J Cell Biol 1993;123:455-465.

31. Deschenes M R, Wilson MH. Age-Related Differences in Synaptic Plasticityfollowing Muscle Unloading. J Neurobiol 2003; 57: 246-256.

32. Carrasco DI, and English AW. Neurotrophin $4 / 5$ is required for the normal development of the slow muscle fiber phenotype in the rat soleus. J Exp Biol 2003; 206: 2191-2200.

33. Fry AC. The role of resistance exercise intensity on muscle fibre adaptations. Sports Med 2004; 34 (10): 663-679.

34. Wang XH, and Poo MM. Potentiation of developing synapses by postsynaptic release of neurotrophin-4. Neuron 1997; 19: 825-835.

35. Wells DG, McKechnie BA, Kelkar S, Fallon JR. Neurotrophins regulate agrin-induced postsynaptic differentiation. Proc. Natl. Acad. Sci, 1999; 96: 1112-1117.

36. Walker UA, Schon EA. Neurotrophin-4 is up-regulated in ragged-red fibers associated with pathogenic mitochondrial DNA mutations. Ann Neurol 1998; 43: 536-540.

37. Mantilla CB, Sieck GC. Trophic factor expression in phrenic motor neurons. Respiratory Physiology \& Neurobiology 2008; 164:252-262.

38. Simon M, Porter R, Brown R, Coulton GR, Terenghi G. Effect of NT-4 and BDNF delivery to damaged sciatic nerves on phenotypic recovery of fast and slow muscles fibres. Eur. J. Neurosci 2003; 18: 2460-2466.

39. Barbacid M. The Trk family of neurotrophin receptors. J Neurobiol 1994; 25: $1386-1403$.

40. Bibel M, Hoppe E, Barde YA. Biochemical and functional interactions between the neurotrophin receptors trk and p75NTR. EMBO J 1999;18: 616-622.

Eslami, R., Gharakhanlou, R., Parnow, A. (2018). Ann Appl Sport Sci, 6(2): 45-53. 
41. Mousavi K, Jasmin BJ. BDNF is expressed in skeletal muscle satellite cells and inhibits myogenic differentiation. J Neurosci 2006; 26: 5739-5749.

42. Deponti D, Buono R, Catanzaro G, Palma CD, Longhi R, Meneveri R, et al. The Low-Affinity Receptor for Neurotrophins p75NTR Plays a Key Role for Satellite Cell Function in Muscle Repair Acting via RhoA. Molecular Biology of the Cell 2009; 20: 3620-3627.

43. Colombo E, Romaggi S, Blasevich F, Mora M, Falcone C, Lochmüller H, et al. The neurotrophin receptor p75NTR is induced on mature myofibres in inflammatory myopathies and promotes myotube survival to inflammatory stress. Neuropathology and Applied Neurobiology 2012; 38(4): 367-378.

Eslami, R., Gharakhanlou, R., Parnow, A. (2018). Ann Appl Sport Sci, 6(2): 45-53. 